\title{
Classification Model of Heart Transplant Outcomes Based on Features of Left Ventricular Functional Geometry
}

\author{
Tatiana Chumarnaya ${ }^{1,2,3}$, Alexander Bykov ${ }^{4}$, Eduard M Idov ${ }^{3,4}$, Sergey P Mikhaylov ${ }^{3,4}$, Olga \\ Solovyova ${ }^{1,2}$ \\ ${ }^{1}$ Institute of Immunology and Physiology, Ural Branch of Russian Academy of Sciences \\ ${ }^{2}$ Ural Federal University \\ ${ }^{3}$ Urals State Medical University \\ ${ }^{4}$ Yekaterinburg Regional Clinical Hospital\#1, Yekaterinburg, Russian Federation
}

\begin{abstract}
The function of the transplanted heart can be significantly affected by acute allograft rejection, chronic rejection, high blood pressure. These factors may induce cardiac remodelling with further adverse consequences for the patients. Dynamic change in the configuration of the left ventricle $(L V)$ from end diastole to end systole ( $L V$ functional geometry) is an important factor of the heart pump function. The objective of this study is to evaluate the time dependent changes in parameters of $L V$ functional geometry in the transplanted heart and to assess relations between the changes and adverse outcomes of the heart transplantation (HT). We used linear discriminant analysis (LDA) to build classification models based on either the standard echocardiographic parameters of LV systolic function and global longitudinal strain (GLS) or LV function geometry indexes. The training set for model building included data from patients with different degrees of systolic dysfunction. Using the models, we retrospectively classified data from 31 patients after orthotropic HT. In contrast to the LDA models based on the standard echocardiographic characteristics and GLS, the model based on the LV functional geometry data showed high accuracy in predicting allograft rejection and development of the heart failure in the HT patients.
\end{abstract}

\section{Introduction}

Heart transplantation (HT) is still the only possible life-saving treatment for the end-stage heart failure, the critical epilogue of several cardiac diseases.

Rejection, especially when associated with hemodynamic compromise, chronic rejection and coronary artery vasculopathy (CAV) remain leading causes of morbidity and graft loss [1]. Therefore, improved detection of adverse changes in the graft function that could predict early acute graft rejection and $\mathrm{CAV}$ is critical for this challenging group of patients $[1,2]$.

Currently the "gold standard" for the diagnosis of allograft rejection and $\mathrm{CAV}$ is cardiac catheterization with endomyocardial biopsy and selective coronary angiography, respectively. This procedure is invasive, carries inherent risk, and may underestimate the deterioration in the graft state. Patients can be evaluated less invasively with echocardiography and clinical examination. The use of noninvasive techniques to assess cardiac allograft function remains a high priority for HT professionals $[2,3]$.

Dynamic changes in the left ventricular (LV) configuration from end diastole to end systole contribute essentially for the effective heart pumping [4]. These changes are termed below "functional geometry" of the LV [5]. It was demonstrated that the coordination of spatio-temporal movements of LV wall segments may be significantly affected by cardiac diseases and by molecular and cellular myocardial remodelling [6].

The aim of this study was to evaluate the time dependent changes in parameters of $\mathrm{LV}$ functional geometry in the transplanted heart by the analysis of echocardiographic LV images and to test the hypothesis that the changes can be used as early markers of allograft dysfunction in the HT patients.

\section{Material and methods}

\subsection{Study population}

We retrospectively examined data from 31 patients after orthotopic HT who had postoperative period from 1 to 9 years. 20 patients who had postoperative period less than 2 years were reviewed for development of acute rejection. The diagnosis of acute rejection was 
Table 1. Indexes of LV

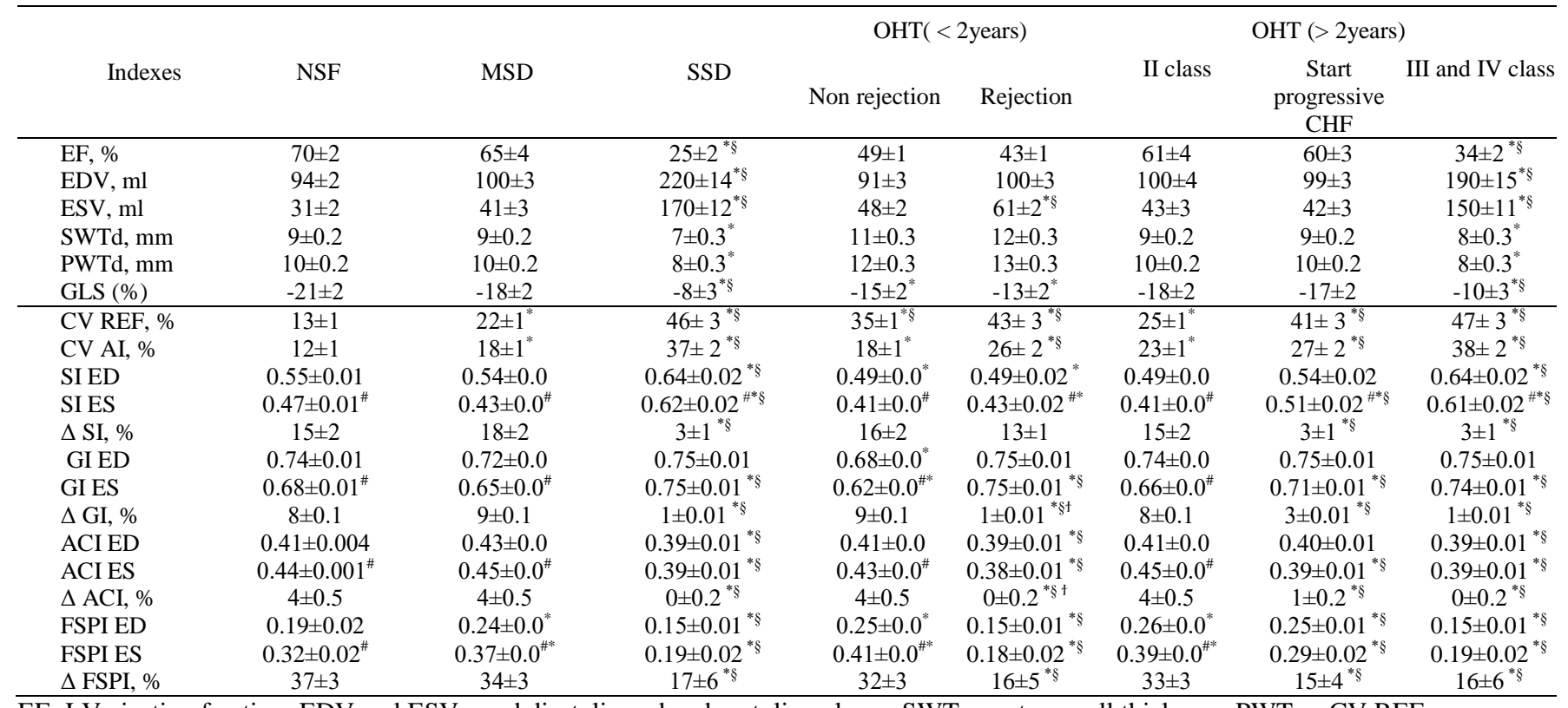

EF- LV ejection fraction, EDV and ESV - end diastolic and end systolic volume, SWT - septum wall thickness, PWT - CV REF -

coefficient of variation of individual regional ejection fraction, CV AI - coefficient of variation of individual asynchrony index, SI sphericity index, ED - end diastole, ES- end systole, $\Delta$ - relative change of index between ED and ES, GI - Gibson index, ACI - apical conicity index, FSPI - Fourier shape-power index, ( \# p<0.05 between ED and ES, ${ }^{*}-\mathrm{p}<0.05$ between pathology and NSF, ${ }^{\S}-\mathrm{p}<0.05$ between SSD and MSD).

based on the criteria of the International Society for Heart and Lung Transplantation (ISHLT). Biopsy specimens with ISHLT grade $2 \mathrm{R}$ rejection or higher were considered as the rejection group and grade $0 \mathrm{R}$ or $1 \mathrm{R}$ were classified as the non-rejection group. There were examined data from data the total 105 cases (4 cases per patient in average) and 38 cases were classified as acute rejection. Biopsy was accompanied by echocardiographic evaluation performed within 1 week before or 1 day after the date of the biopsy.

17 patients had postoperative period more than 2 years (total 67 cases). This subgroup of patients was investigated for progressive chronic heart failure (CHF). In addition to standard clinical procedures for assessing the patient's condition, echocardiography was performed. For 27 cases, class II CHF was identified according to the New York classification of American Heart Association (AHA), for 25 cases III-IV grade of CHF. In 15 cases, echocardiography was evaluated in a month before the adverse outcomes (progression of CHF).

The training set for building classification models consisted of the data from three groups of patients with different degrees of LV systolic dysfunction. The first control group consisted of data from 24 healthy volunteers without signs of cardiovascular diseases called below as the group of normal systolic function (NSF). The second group of mild systolic dysfunction (MSD) with preserved LV systolic function (ejection fraction $>50 \%$ ) consisted of 52 patients suffering from ischemic heart disease. And the third group with significant LV systolic dysfunction (SSD) consisted of 25 patients with dilatational cardiomyopathy (ejection fraction $<35 \%$ ). The study was approved by ethics comities of Yekaterinburg Regional Clinical Hospital \# 1. All subjects provided written informed consent.

\subsection{Echocardiography}

Ultrasound LV images in classical 2D apical fourchamber view were recorded during the entire cardiac cycle simultaneously with ECG recording by ultrasound system Philips IE33. The systolic function was evaluated by Simpson's method. Echocardiography standard protocol involved such parameters as: LV ejection fraction, LV end-diastolic and end-systolic volumes, thicknesses of septum and of the posterior LV wall. In addition, the endocardial and epicardial borders were obtained semiautomatic by using QLab (Philips). These borders were visually inspected throughout the cardiac cycle and manually adjusted if necessary. Peak systolic longitudinal strain was obtained in $7 \mathrm{LV}$ segments from an apical 4-chamber view by using QLab. Global longitudinal strain (GLS) was calculated as the average of strain values obtained from the 7 segments.

For evaluation of functional geometry parameters all echocardographic images with myocardial borders were digitized and analyzed off-line with using custom made software [5]. The contribution of different LV wall 

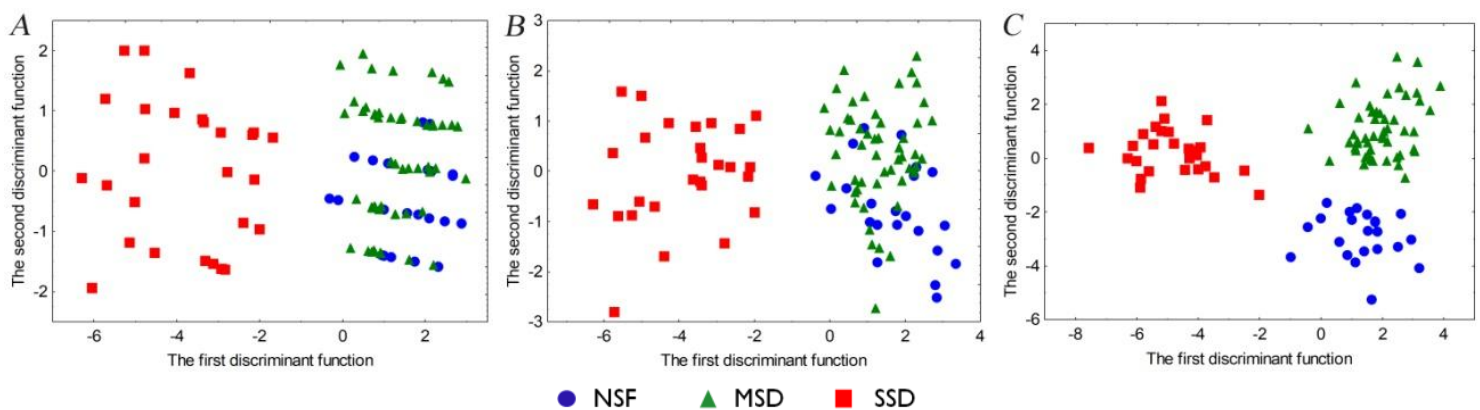

Figure1. LDA classification models. A) Model based on standard protocol. B) Model based on standard protocol and GLS. C) Model based on LV functional geometry parameters.
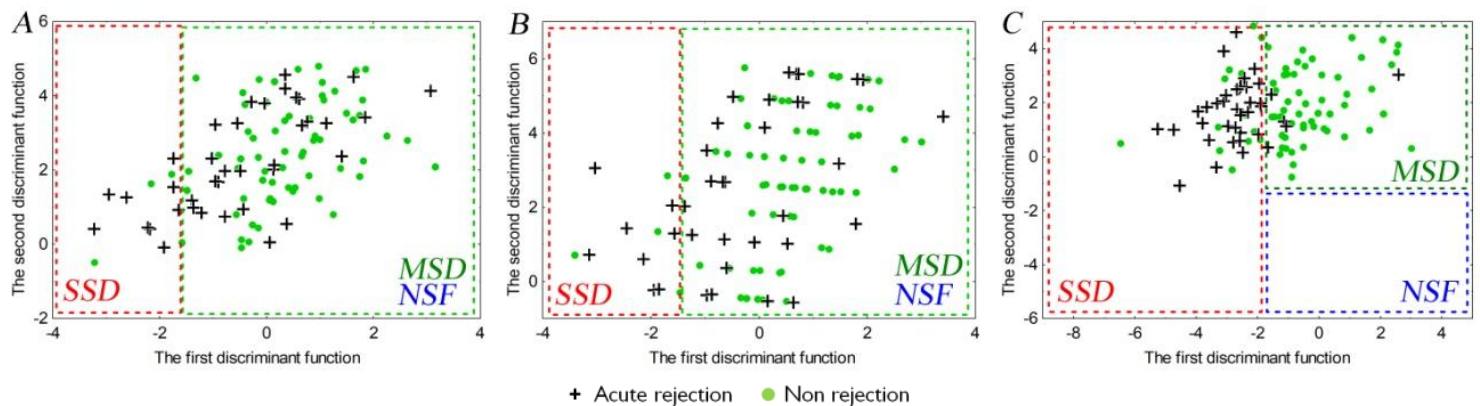

Figure 2. Scatterplot of canonical scores in HT patients which were reviewed for development of acute rejection. A) Standard protocol model; B) standard protocol and GLS model; C) LV functional geometry model.
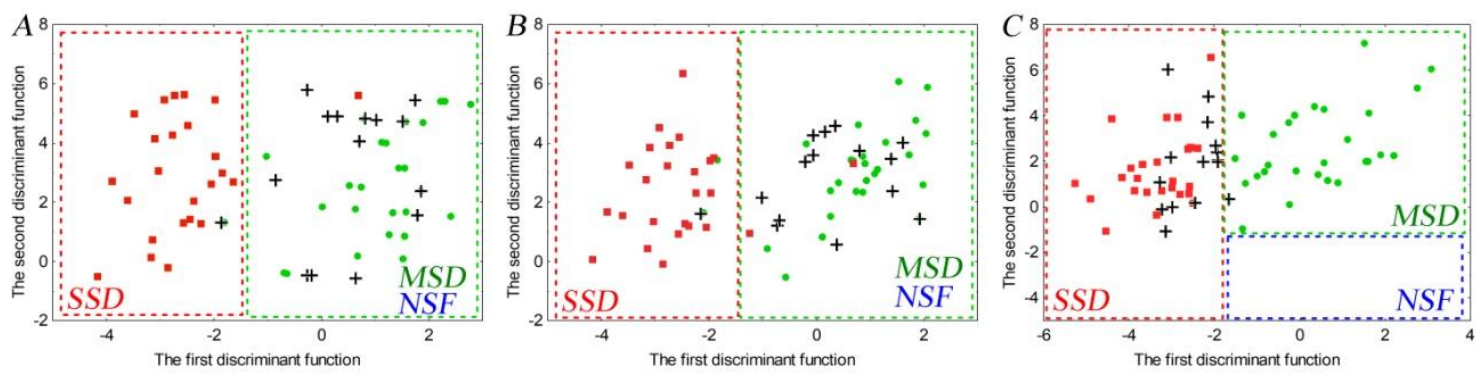

+ Progressive $\mathrm{CHF}$ - II class $\mathrm{CHF} \| \mathrm{III}-\mathrm{IV}$ class $\mathrm{CHF}$

Figure 3. Scatterplot of canonical scores in HT patients which were investigated for progressive CHF. A) Standard protocol model; B) standard protocol and GLS model; C) LV functional geometry model.

regions in global ejection fraction was evaluated by the computerized automatic analysis of the LV wall segmental kinetics. Internal LV sectors were obtained by segmental division of the LV image along the endocardial contour. The regional ejection fractions have been calculated as the maximal changes in sector areas during the cycle. Asynchrony of regional contractions was assessed by the fraction of the time to approach the minimal sector area (local systole) during the cycle in the duration of global systole. The coefficients of variation in the individual regional ejection fractions and in the asynchrony data for a patient were used as individual indexes of spatial and temporal heterogeneity in the LV wall regional function [5]. We assessed also several LV shape indexes: 1) conventional sphericity index; 2) Gibson index, which also characterizes a degree of shape circularity; 3) apical conicity index which shows the degree of conical shape of the apical zone; 4) Fourier shape-power index showing the complexity of the LV shape as compared to the circle $[5,6]$.

\subsection{Classification model}

We used linear discriminant analysis (LDA) to build classification models for evaluating state of transplant allograft. The first model was based on parameters from standard protocol (Table 1). The second model used standard echocardiography parameters and GLS. The third classification model was based on only LV function geometry indexes without using standard protocol characteristics(Table 1).

Statistical analysis was performed by SPSS 22.0. All 
shown data are expressed as Means \pm Standard Deviation for the entire group of subjects. A P value of $<0.05$ was considered statistically significant.

\section{Results}

In the previous work [5] we investigated the differences in the parameters of LV functional geometry between groups of patients with different degrees of LV systolic dysfunction. The characteristics of the LV functional geometry in NSF, MSD, SSD groups used to build the classification models are shown in Table 1.

Using LDA, we built three classification models based on training set data from NSF, MSD and SSD patient groups. The first model used data from the standard echocardiography protocol and showed the classification accuracy of $13 \%$ for NSF, $96 \%$ for MSD, and $100 \%$ for SSD (Fig. 1A). So, this model was not able to separate the NSF and MSD groups. When in addition to conventional echocardiographic parameters the GLS values were accounted for in the first model, the quality of the classification improved, showing accuracy of $45 \%$ for NSF, $90 \%$ for MSD, and $100 \%$ for SSF. But this model also could not clearly distinguish MSD from NSF group (Fig 1B). The third model built on the characteristics of the LV functional geometry, stratified data from all the three groups with an accuracy of $100 \%$ (Fig. 1C). This model clearly delineated data between the NSF and MSD groups.

\section{Stratification of HT patients}

Each of the three LDA models was used to classify data from HT patients with postoperative period less than 2 years in terms of the possibility to predict the acute allograft rejection. The first model based on the standard echocardiographic data and the second model based on the latter plus GLS classified $21 \%$ and $24 \%$ of acute rejection cases in SSD group and the rest cases in MSD group respectively (Fig. $2 \mathrm{~A}, \mathrm{~B}$ ). Thus, these two models failed in stratification of the data between the allograph rejection group and other HT cases. The third model built on the LV functional geometry data classified $90 \%$ of cases with acute rejection in the SSD group (Fig. 2 C), showing a high accuracy in data stratification and high power in prediction for the acute allograph rejection.

Data from HT patients with a postoperative period of more than 2 years was classified using the LDA models in terms of the possibility to predict progression of $\mathrm{CHF}$. All the models demonstrated a high accuracy in data stratification according to the AHA CHF classification. More than $90 \%$ cases from class II patients were classified in MSD group and more than 90\% cases from class III, IV patients were classified in SSD group. The highest accuracy of $100 \%$ was demonstrated by the third LDA model based on the LV functional geometry data (Fig. 3). Then, we used the LDA models to classify data from 15 patients which were evaluated within a month before deterioration of their state and confirmed $\mathrm{CHF}$ progression with reclassification at upper CHF class. Models based on the standard echocardiographic characteristics and GLS classified only 1 of the 15 cases in the SSD group while the rest data were classified in MSD. In contrast, the model based on the functional geometry data classified 14 of the 15 cases in SSD group predicting $\mathrm{CHF}$ progression before adverse outcomes (Fig. 3).

\section{Conclusion}

Our LDA model built on the parameters of LV functional geometry derived from the conventional LV echocardiographic images shows a high power in predicting for acute allograph rejection and for adverse outcomes of CHF progression in HT patients.

\section{Acknowledgements}

This work was supported by RF Government Act \#211 of March 16, 2013 (agreement 02.A03.21.0006. The work was carried out within the framework of the IIF UrB RAS theme No AAAA-A18-118020590031-8.

\section{References}

[1] Sabia C., Picascia A., Grimaldi V., Amarelli C., Maiello C., Napoli C. The epigenetic promise to improve prognosis of heart failure and heart transplantation. Transplantation Reviews. 2017;31:249-256.

[2] Alraies M.C., Eckman P. Adult heart transplant: indications and outcomes. Journal of thoracic disease. 2014; 6(8):11201128.

[3] Mangini S., Alves B.R., Silvestre O.M., Pires P.V., Pires L.J.T., Curiati M.N.C., Bacal F. Heart transplantation. 2015; 13(2):310-318.

[4] Sengupta P.P., Korinek J., Belohlavek M., Narula J., Vannan M.A., Jahangir A., Khandheria B.K. Left ventricular structure and function: basic science for cardiac imaging. Journal of the American College of Cardiology. 2006; 48(10):1988-2001.

[5] Chumarnaya T., Alueva Y.S., Kochmasheva V., Mihailov S., Revishvili A.S., Tsyv'ian P., Solov'eva O. Features of the Left ventricular functional geometry in patients with myocardial diseases with varying degrees of systolic dysfunction. Bulletin of experimental biology and medicine. 2016; 162(1):30-34.

[6] Markhasin V.S., Solovyova O., Chumarnaya T.V., Sukhareva S.V. Problem of heterogeneity of myocardial. Russian Journal of Physiology. 2009; 95(9):919-943.

Address for correspondence.

Tatiana Chumarnaya.

Pervomaiskaya str. 106, Ekaterinburg, Russia 620002.

chumarnaya@gmail.com. 